\title{
Online-Computer-Mediated Interviews and Observations: Overcoming Challenges and Establishing Best Practices in a Human-AI Teaming Context
}

\author{
Keri K. Stephens \\ The University of Texas at Austin \\ keristephens@austin.utexas.edu \\ Carolyn E. Montagnolo \\ The University of Texas at Austin \\ cmontagnolo@utexas.edu
}

\author{
Karim Nader \\ The University of Texas at Austin \\ karim.nader@utexas.edu
}

Amanda L. Hughes

Brigham Young University

Amanda_Hughes@byu.edu

\author{
Anastazja G. Harris \\ The University of Texas at Austin \\ harrisana@utexas.edu \\ S. Ashley Jarvis \\ Brigham Young University \\ sashleystevens3@gmail.com
}

\author{
Yasas Senarath \\ George Mason University \\ ywijesu@gmu.edu
}

\author{
Hemant Purohit \\ George Mason University \\ hpurohit@gmu.edu
}

\begin{abstract}
Prior research has established the feasibility of conducting online interviews and observations, yet there is limited guidance in how to interact with participants when conducting fully mediated research with screen-sharing and video. This study, conducted during early phases of COVID-19, included 15 volunteer tweet-annotators working with an emergency response organization. This method contribution uses cues-related and surveillance theories to reveal challenges and best practices when asking research participants to share their screen, be on video, and participate in a multiple-interview study. The findings suggest that researchers conducting online-mediated research should be prepared to provide technical support for the devices and interfaces participants use during the study, find ways to "see" beyond what is on the mediated screen, and consider ethical issues not often discussed. In addition to these findings, an output of this research is two brief training videos useful for other researchers embarking on conducting fully mediated research.
\end{abstract}

\section{Introduction}

COVID-19 forced qualitative researchers around the globe to shift gears quickly and collect data in a mediated context due to stay-at-home orders and social distancing $[1,2]$. Travel restrictions affected data collection in our multiple-method research project that involved interviewing and observing human annotators while they worked with a computer interface for data filtering that used machine learning in a human-AI teaming context. Therefore, we documented our procedures, made rapid adjustments, and identified successful processes we used to collect over fifty online interviews in under two months.

The main contribution of this work is to demonstrate how to conduct mediated, synchronous data collection, and specify how this differs from inperson methods. To establish the unique contribution of this method-focused paper, we ground our research in the current literature describing how to conduct online interviews and observations $[3,4,5]$. We use cues-filtered-out related theories $[6,7,8]$ to justify the need to conduct multiple interviews with the same participant, something rarely done in mediated research. These theories also guide the development of a research question to address the unique, and thus contributing aspects of this method-focused paper. After describing our method, we share the major findings in the form of challenges that emerged during data collection. We end by elaborating theoretical contributions and establishing best practices for conducting fully mediated multiple-method research. 


\section{Conducting Research Online}

The Sage Handbook of Online Research Methods [9], offers resources on the topic of online research (e.g., [5]), and describes specific approaches to collecting data online. Although, it was published fairly recently in 2017 , there is limited discussion of conducting fully-online interview and observational studies. Our focus here centers on this multiplemethod approach, and how it differs from in-person research. First, we review theories that help explain these differences.

\subsection{Theoretical explanations}

On the surface, it appears that moving interviews and observations online is simply a logistical difference from conducting them in-person, but past theoretical work on cues-filtered out approaches suggests relationship quality can suffer because of the lack of nonverbal cues found in mediated contexts [6]. Even video conversations lack the perceived richness of face-to-face [10], due in part, to the fact that subtle nonverbal cues are missing when conversations are mediated, and events happen outside the view of the video cameras $[6,11]$. Thus, it can be hard to build rapport or gain trust in mediated contexts due to the lack of visual and physical cues [12].

However, building on the cues-filtered-out perspective, Walther developed the hyperpersonal model, because he found that over time, with multiple interactions, even in mediated contexts, relationships can become as strong as face-to-face [7, 8]. Empirical work in online interviewing using Skype has applied Walther's work $[7,8]$ to try to overcome the lack of cues by exchanging several emails prior to conducting online interviews [13].

2.1.2 Surveillance. Another difference between online and in-person observations concerns the degree to which people feel watched. The theoretical work on surveillance provides insight into how specific aspects of mediated research might contribute to a panoptic effect above and beyond what would happen in person $[13,14]$. Specifically, theoretical work around the panopticon discusses how the watcher can see those being watched, while those being watched can't see who is watching them [14]. Depending on how video cameras are configured, this can be an issue in mediated contexts. Additionally, technology competence can make people feel more self-conscious about being watched. Specifically, the more frequently an individual uses technology-like online platforms - the more comfortable they are, the more proficient they become, and the less they feel like they are being surveilled [15]. Online research requires participants to use technology to participate in the interview; something that does not happen in person. Therefore, people less comfortable with technology used for online interviews will likely feel more surveilled.

\subsection{Synchronous online interviewing research}

Interviews online can happen in two predominant ways: synchronously or asynchronously [9]. Historically, much of the research around online interviews has followed an asynchronous form, such as using email exchanges [16, 17, 18, 19], because these approaches are more time efficient. With synchronous interview methods, researchers who want to preserve convenience and accessibility can opt for instant messaging methods [20,21], or phone-based calls [12]. However, using video for research is becoming more common. This method is particularly relevant at this time since many people transitioned to working from home because of the spread of COVID19 [1]. To date, most of the existing research using online video interviewing has used the platform, Skype, to conduct interviews and sometimes observations as well [4, 13, 22, 23, 24].

There is almost no mention of conducting observations or directly interacting with participants through sharing screens in the existing research method literature. The exceptions for online observations fall primarily within the study of virtual worlds like World of Warcraft or Second Life (e.g., [25]), but these researchers are often acting as participant observers, not simply watching a participant complete a task. Observing via screen sharing is not mentioned in the literature, most likely because it is a more recently available feature; but it is important to study because it allows a unique form of interaction between interviewer and interviewee. To characterize the available literature on mediated research, next we describe advantages and challenges scholars have identified with synchronous online interviewing and observations.

2.2.1 Advantages. Quite often, there are logistical advantages for both the interviewer and interviewee when conducting interviews online, for example, a lack of travel costs [12, 20, 22]. Another advantage is the broad range of times available to conduct the interviews. For example, it is easier to work around interviewees' work schedules by interviewing them during evenings and weekends; something less desirable when meeting in person $[13,20]$. This also means that more people can participate in this type of study, which could improve the quality of the sample 
[13]. Additionally, many video conferencing platforms generate transcripts automatically, reducing the cost of having data transcribed [21].

2.2.2 Disadvantages. Prior research suggests there are several disadvantages as well. Shared technology is essential during online interviews and observations, and some participants may not have stable internet access, causing technical problems [24]. Technological and online competency is also a consideration since interviewees must be able to successfully navigate the computer interface. While not possible in all studies, some studies have let their interviewees choose the platform they prefer to use [20], as one way to help them have more competence with the tools used for data collection. Yet, only selecting participants with strong technological competency is also a drawback since it can increase sample homogeneity.

Another type of disadvantage relates to the cuesfiltered-out perspective [6]. For example, body language is limited in an online reduced-cues environment, so interviewers can miss visual and physical cues necessary to help build rapport [12]. Furthermore, researchers have no control over distractions present in the interviewees environment [21]. For example, interviewees may become distracted when they multitask while also trying to participate in the research study [21]. Finally, prior research has found that in mediated contexts interviewers have to learn how to give verbal and textual feedback during the interview, and it can be hard to keep their interviewee engaged in sharing meaningful information [21].

Even though past research has provided nice grounding in advantages and disadvantages of using synchronous online interviews and observations, there are still some prominent gaps. First, there is a dearth of research on how to conduct multiple interviews and observations with the same participants over time, and theories suggest this is important in reduced cues environments. Additionally, there is no research on collecting data by asking participants to share their screen. Considering the growing prominence of these strategies, we ask the first research question:

RQ1: When conducting synchronous mediated research where participants interact with researchers over multiple interviews, what challenges emerge?

\subsection{Identifying Best Practices}

Considering that in-person interviews and observations were suspended, or delayed, due to the spread of COVID-19, it is important to chart a meaningful course of action for qualitative research to be conducted online now and in the future [1]. While past research has identified some advantages of these methods, we know very little about best practices that can be shared. Therefore, we ask the following research question:

RQ2: How can we convert learned best practices around synchronous mediated research into training for other researchers?

\section{Method}

Prior to receiving funding to study human-AI teaming during COVID-19, the researchers reviewed existing literature to establish a protocol for observing and interviewing participants as they label data. There were researchers from three different academic institutions, as well as the manager of the Virtual Emergency Response Team (VERT), who trained and facilitated access to the study volunteers. In addition, there was an online annotation platform that the volunteers used to label COVID-19-related tweets, and a key part of the overall research project involved observing this annotation process; therefore, screen sharing was essential. All three institutions involved had access to Zoom, and that was the platform used to train the volunteers, so this tool was chosen as the data collection platform. Zoom could handle all the requirements of this study: ease of access (free accounts), camera availability, ability to screen share, and the capability to record and automatically transcribe the interview sessions.

\subsection{Participants}

Participants in this study included 14 (VERT) volunteers and one manager (See Table 1). They were recruited by the manager and all had prior experience annotating tweets during COVID-19. In this study, thirteen of the VERT volunteers participated in three separate one-hour interview/observation sessions and one volunteer participated in two separate sessions. The volunteers were paid $\$ 25 /$ hour for a total of $\$ 75$ for participating in the three phases of data collection. The manager participated in two longer sessions, and since he is paid by the federal government, he was not financially compensated for his participation.

Table 1 describes each participant, the technology they used to participate in the research, their age, and specific area of expertise. Several participants were experienced annotators who had volunteered during disasters prior to COVID-19. Considering the focus 
of the larger research project is to understand the interactions between humans and machines, technology-specific and social-media-specific expertise was also captured.

\section{Table 1: Participant information}

\begin{tabular}{|c|c|c|c|}
\hline ID & $\begin{array}{l}\text { TECH } \\
\text { USED }\end{array}$ & AGE & $\begin{array}{l}\text { TASK-RELEVANT } \\
\text { EXPERTISE }\end{array}$ \\
\hline $01 *$ & $\mathrm{PC}$ & 52 & Emergency manager \\
\hline 02 & iPad & 46 & $\begin{array}{l}\text { Works in IT; experienced } \\
\text { annotator }\end{array}$ \\
\hline 03 & $\mathrm{PC}$ & 71 & Former emergency manager \\
\hline 04 & Mac & $\begin{array}{l}\text { Late } \\
30 \mathrm{~s}\end{array}$ & $\begin{array}{l}\text { Experienced annotator; } \\
\text { emergency manager; Twitter }\end{array}$ \\
\hline 05 & $\mathrm{PC}$ & 73 & CERT Volunteer \\
\hline 06 & $\mathrm{PC}$ & 44 & $\begin{array}{l}\text { Experienced annotator; Works } \\
\text { in IT; ML; Twitter }\end{array}$ \\
\hline 07 & $\mathrm{PC}$ & 66 & NLP experience \\
\hline 08 & iPad & $\begin{array}{l}\text { Late } \\
30 \mathrm{~s}\end{array}$ & $\begin{array}{l}\text { Experienced annotator; Works } \\
\text { in IT; NLP; Twitter \& social } \\
\text { media }\end{array}$ \\
\hline 09 & $\mathrm{PC}$ & 37 & $\begin{array}{l}\text { Experienced annotator; data } \\
\text { mining; Twitter \& social } \\
\text { media }\end{array}$ \\
\hline 10 & Mac & 31 & Social media (Facebook) \\
\hline 11 & Mac & 70 & Experienced annotator \\
\hline 12 & $\mathrm{PC}$ & 68 & Social media (Facebook) \\
\hline 13 & Mac & 39 & Twitter \& social media \\
\hline 14 & $\mathrm{PC}$ & 53 & $\begin{array}{l}\text { Experienced annotator; } \\
\text { Twitter \& other social media }\end{array}$ \\
\hline 15 & $\mathrm{PC}$ & 49 & Experienced annotator \\
\hline
\end{tabular}

\subsection{Data collection}

A total of five different researchers collected data from the volunteers. In all but two situations, at least two researchers were present during each online session, and each researcher took field notes related to the content of the annotation study, as well as observations around the process of conducting online synchronous data. Interviews were scheduled through email correspondence or through Calendly, an appointment scheduling software that allows users to create specific timeslots that others can book. Calendly provided an advantage over traditional email scheduling since it prevented participants from requesting interview times that would not fit into interviewers' schedules, thereby cutting down on email exchanges. Additionally, the automated scheduling of Calendly sent calendar invites to the interviewer and interviewee in addition to blocking out that time on the interviewer's calendar so other interviewees could not reserve that time if it was already filled.
A semi-structured interview schedule guided the data collection, and the project was IRB approved. A think-aloud protocol-a well-established approach that asks participants who are performing a task to talk through the decisions they are making as they perform the task [26] — was used during the interviews. While labeling tweets, researchers provided non-directive, yet supportive feedback, and periodically asked the volunteer to provide more explanation for why they chose specific annotation labels.

\subsection{Data analysis}

Data from the online observation and interview sessions were video recorded and subsequently transcribed. The transcripts were automatically generated by Zoom and included timestamps so they could be used as subtitles for the video file.

To address the method study's research questions, the five different researchers met weekly for eight weeks to identify data collection challenges and implement changes. We relied on the field notes each researcher had each taken as they documented method challenges and best practices, reviewed video tapes, and discussed our research process observations. The principles guiding our analysis included reviewing the prior theoretical and empirical work that informed the study, and identifying the challenges, workarounds, and best practices that emerged during data analysis. We balanced our knowledge of the literature with our goal to maintain openness as we allowed new findings to emerge. We generated themes that addressed the two research questions, and used a constant comparative analysis [27] to collapse similar codes into core categories. A minimum of two researchers reviewed each category to verify the systematic approach used.

\section{Findings}

\subsection{RQ1: Challenges in mediated research}

The first research question asked about the challenges that emerge when conducting multiple interviews with the same respondent using fully mediated research where participants interact with the researchers. We identified five core categories that reinforced findings uncovered during our literature review. Specifically, there were technical issues, competence with technology concerns, rapportbuilding issues, distractions in both the researcher's and interviewee's environments, and challenges in making decisions around how to provide reinforcing feedback (called listening in prior research [21]). By 
conducting multiple interviews, sharing screens in every interview, and observing the participants annotating tweets, we identified five major categories that are unique: 1) confusion around using different technologies forced researchers to become real-time technology help desks, 2) privacy concerns arose that were unique to online interviewing, 3) the mediated context meant the researchers could not see the whole picture, 4) think aloud protocols are particularly challenging online, and 5) observational concerns are much more complex than in-person. Taken together, these findings illustrate key differences between conducting interviews and observations in-person and online.

4.1.1. Researchers as help desks. There was confusion surrounding technology use that forced the researchers to act as real-time help desks. Multiple times the research team "thought" they were using the same technology-Zoom-but there were actually many differences that surfaced during data collection. First, the institutional licenses of Zoom contained different settings, so with participants from three different institutions, the rules for using the same product, Zoom, were different. For example, one institution had experienced Zoombombing where unauthorized people attended sessions and were inappropriate. This institution was actively managing the security and participation settings inside their licensed product and that made it challenging for anyone outside the organization to be a part of a Zoom event hosted by a member of The University of Texas at Austin. This was a challenge for the research team and study participants, who had to enter sessions as guests through a virtual waiting room.

Zoom account differences were especially challenging for study participants. Even though they had all participated in the annotator training hosted by their manager via Zoom, they did not all have personal or work Zoom accounts; they joined Zoom using single sign-ons like Facebook or their Google account. At first, the research team was unaware that the security settings for one institution required all participants to enter through a Zoom account. Once we learned about these issues, we included this detail in the email inviting participants to join the online data session. However, people did not read that instruction, so they were blocked. As a result, there were occasions when the time it took for participants to access the Zoom meeting took a significant amount of time away from the research-specific interview conversation and observations, especially during the first of the three interviewees.

Properly configuring remote video and screen share with study participants, is much more complex than being in-person conducting interviews and observations. The participants used different computing and mobile devices to participate in the study. For example, Interviewee \#04 used Zoom on her mobile phone, and was unable to screenshare as she was running the annotating program on her PC. She was very computer savvy, so she quickly hung up her phone, configured her computer for Zoom, and logged back into the session. This took less than five minutes but could have been more challenging for a less experienced participant.

Another participant, Interviewee \#05 joined the Zoom call, and when asked to screenshare could not find the screenshare buttons. After a 5-minute conversation, she said she was using an iPad, and one of the interviewers then found online instructions to walk her through how to use an iPad and screen share. A major point of confusion in this case was that the buttons were called different things ("Share Screen" on a desktop, and "Share Content" on an iPad).

One participant had such serious bandwidth issues that he was bumped off the session seven times even after turning off all video. Finally, four interviewees either lost their passwords or could not find the correct link to the data they needed to annotate. All these challenges meant that researchers played the dual role of technology support and interviewer/observer.

Several interviewers were in their early 20's, and they felt nervous about seeming bossy to interviewees when deciding whether to jump in and assume a helpdesk role. This role included guiding participants on various tasks related to using the necessary technology such as providing instructions for screen sharing. Occasionally interviewees became stressed when trying to figure how to use the technology correctly, so researchers had to be careful when jumping in to help so as to not exacerbate the stress. The researchers wanted to be supportive and help reduce the interviewees frustration, but they had a limited view of what was happening on the interviewees' sides of the conversation.

When participants were experiencing technology issues, it was helpful to have two interviewers work to address the problem. In some cases, interviewers took advantage of Zoom's private chat feature to communicate with each other about the best way to help the participant. Additionally, there were times when one interviewer maintained communication with the participant while the other searched for resources to help solve the problem. These methods allowed the team to resolve issues more swiftly than they could have individually, and kept participants engaged during these interruptions. 
4.1.2. Privacy concerns. Recording Zoom sessions revealed several privacy concerns to manage. After the first interview, the researchers realized that in Zoom video recordings and transcripts, participants' names are recorded. To address this the researchers edited this first video and transcript manually, but in future interviews, prior to recording the session, the researchers replaced participants' names with a subject number using features available in Zoom.

Other privacy concerns stemmed from asking participants to share their screens. Occasionally, information outside the context of the study become visible on the participants' screens. For example, while trying to navigate to the correct annotation task, one participant opened her personal email account. Additionally, several personal text messages momentarily appeared on participants' screens. Getting glimpses of private information is less likely to occur when interviews occur in person.

Since the Zoom sessions took place in participants' homes, other members of their household would sometimes appear visually on the video or they could be heard through the video's audio. These appearances were fleeting and innocuous but raise questions about the privacy of others in the study environment who have not agreed to participate in the research. What if they reveal sensitive information (visually or verbally) about themselves or the participant? Such challenges have always existed for researchers who interview or observe people in their homes. However, when researchers are physically present in the home environment (often with cameras and video equipment) it is clearer that data about anyone in the room might be captured. These cues are absent —or at the least far less visible - when using online video to interact with a study participant.

4.1.3. Researchers missing context. Attempting to conduct interactive interviews while located remotely revealed several challenges that also demonstrate how online differs from in-person. For example, researchers were not aware of the weather forecasts and conditions at the participants' location. Around one third of the interviews were conducted while thunderstorms occurred in the interviewees' locations, which introduced concerns around having bandwidth and power issues that would prevent completing the sessions.

The inability to see the whole picture (i.e., what was off camera) meant the researchers had to actively plan questions to learn about the annotator's environment. For example, through questions we learned three of the participants printed the definitions of their labeling rules and sat them on their desk or pinned them to a wall for quick reference. Researchers also could not see the technology the interviewees were using, and this contributed to some of the technology-specific challenges described in 4.1.1.

\subsubsection{Think aloud challenges.}

Think aloud protocols ask respondents to verbalize their thinking process, and they are commonly used to understand the decisions behind a person's actions. Our findings suggest that this approach may be more challenging online than if conducted in person, although this challenge diminishes when more than one interview is conducted. The researchers can see both the screen and the camera feed of the participants as they read the tweet and verbalize their labeling decision. This process seemed more involved in a mediated context than if the interviewer had been out of view of the annotator and simply looking over their shoulder. Some of the participants wanted affirmation that they were performing their task well and would frequently ask the researchers' opinions. It was as if they viewed the researcher as a collaborator because the faces of both parties were on the computer screen. This meant that the researchers had to provide verbal and nonverbal feedback, while consciously trying to not affect the annotation outcome. Being "in their face," literally, likely also reminded them they were being watched.

4.1.5 Observation effects. There were several codes in the data that we collapsed in this observation effect category that centered around camera issues and panopticon effects. The researchers found that having a camera became a focal point, at certain times, during interviews. At the start of the interviews, before screen sharing, the camera feeds of all participants took up the entirety of the participant's screen. The participants would move their cameras around to be sure to talk directly to the interviewer, and they would also see themselves on their screen and comment on their own appearance. Participant \#03 noticed her physical appearance on her video feed in the Zoom interface and quickly fixed her hair while commenting on it before continuing with the interview. Those interactions made the researchers aware of the panoptic effect: being on the screen of the participants enhanced their awareness that they were being observed and surveilled, and sometimes they changed their behavior because of that.

However, when participants start sharing their screen, the Zoom interface and the camera feed are both minimized. The interface, along with any indication of being observed and recorded, is minimized and the participant's screen looks as if they are working alone. While many of these settings are adjustable inside of individual preferences in Zoom, 
most commonly Zoom's minimized camera feed shows the active participant, meaning the participant who is talking at the time. Since the interviewer was muted to allow the participants to share their thoughts, the participants would rarely see the interviewer's camera feed or hear verbal cues from the interviewer. With little visual and auditory reminders that they are being observed, the participant may be under a more serious panoptic effect than during an in-person interview. This is because only the interviewee shared their screen, so this was a one-way observation, something literature on the panopticon says can be quite disconcerting.

To reduce the panoptic effect in this mediated research study, the researchers always shared their camera feed so the participants would not feel as though they were seen by others but unable to see the observer [15]. Another way the researchers tried to reduce the panoptic effect was to use software familiar to the participant. Zoom was widely adopted in the U.S. at the start of the COVID-19 pandemic, and most participants were familiar with the software and the interface since they had used Zoom in both social and professional contexts. However, as previously discussed in 4.1.1., thinking we had the same technology tools was not often an accurate assumption.

The researchers also struggled with how to effectively work with the camera and mute button, and this was related to observation effects. Researchers knew that looking directly into the camera was the only way the interviewee would see them maintaining eye contact, because if they looked down at their screen, or watched the face of the interviewee, it would appear they were not looking into their eyes. They felt added pressure to overcome the reduced-cues environment and pay attention to the participants, so they recalled spending time trying to look meaningfully into the camera to maintain eye contact. However, they also acknowledged, that the participants were often engaged in their annotating task, so it was not clear that that attention to the camera was worth the potential of missing out on meaningful nonverbal behaviors of the annotators. The fact that using a camera means we cannot both look like we are maintaining eye contact, and actually look at the person on the screen, is a serious challenge present in online interviewing. In addition, researchers often used the mute button to reduce feedback and eliminate sounds in their own environment. This meant that when an interviewee made a comment, we were providing many fewer verbal reinforcements than would have occurred in person.

\subsection{RQ2: Convert Learning for IS Field}

The final research question asked how to convert learned best practices around mediated research into training for other researchers. To address this question, we reviewed our research findings and used them to create a practical outcome of this project: two brief training videos that captured best practices. While these videos are deliberately brief, one of them is presented in a question and answer format, and the other is a lecture format with slides. These videos can be accessed here https://youtu.be/g1pW-BubO88, https://youtu.be/nT_D9A6gz4M and they focus on 1) how to set your team up for success when conducting online interviews with screen sharing, and 2) how to design an online mediated research project when the goal is to maximize interaction (including screen sharing). We discuss these more fully in the discussion.

\section{Discussion}

This method contribution moves beyond a basic understanding of how to conduct interviews and observations online and focuses on the challenges of interacting with research subjects through fully mediated environments. It also illustrates that even though online and in-person observations and interviews are both conducted synchronously, and you can see one another, moving online requires considerable understanding of challenges found in mediated contexts. The key findings suggest there are many more nuanced considerations around planning and executing synchronous online data collection efforts than have been identified in the past.

\subsection{Theoretical Contributions}

By using the knowledge of prior $\mathrm{CMC}$ researchers and scholars conducting online interviews [7, 8, 13], our team designed a solid, theory-informed approach to strengthen trust and build rapport. Specifically, we designed our data collection to compensate for negative computer-mediated effects by including multiple interactions with the interviewees over time.

While we certainly saw evidence in our data that theories related to cues-filtered-out [6] explain portions of our findings concerning human factors, we also found evidence to support the hyperpersonal model [8]. Walther describes that in some mediated contexts, the opportunities for selective selfpresentation, idealization, and reciprocation can help people exceed the relationship goals they would have accomplished if face-to-face [8]. Specifically, in our 
study, interviewee \#04 continued the email conversation well beyond the third interview. She sent the lead interviewer an example of what a tweet generated by a bot looked like and explained her reasoning; she also sent a recipe for key lime pie. Interviewee \#13 sent one of the researchers a LinkedIn invitation, and Interviewee \#08 said, "When you are in the DC area, let me know and I'll take you to dinner." Several interviewees commented at the completion of the third interview that they were sorry the research was over, and Interviewee \#07 said, "it is so hard to say goodbye since we got to know each other so well." While we have no way of knowing if the same experiences would have happened if the interviews had been conducted in person, the data suggest that strong relationships can form in online research, much like they do, sometimes, in face-to-face interviews.

In addition to extending the applicability of these theories to online mediated communication, the grounded approach to our analysis also allowed another theoretical contribution to emerge around the notion of technology affordances. Affordances are choices people make when they use technologies [28, 29]. While many people confuse affordances and features of technology [30], this study clearly illustrates how people use the same technology differently by drawing upon diverse affordances. Furthermore, what is called the same technologyZoom-can have different features enabled or disabled, creating an added layer of complexity to understanding affordances. We had not anticipated the need to help the interviewees participate in the study, and despite the fact that most of them were technologically literate, the research team had to move beyond discussing technology or features, and instead individualize our communication around how the technology was being used for every user. The findings highlight the importance of the concept of affordances of technology, and how relevant these are when conducting fully mediated research.

\subsection{Online Research Method Contributions}

The most recent SAGE Handbook of Online Research Methods [9] only has a few paragraphs about synchronous interviewing online, and this paper extends this research by sharing details about our methods and key learnings. Several findings discussed here and in the two videos created as a supplement to this research, offer considerable contributions for online research methods. First, this study demonstrates that moving interviews online is not simply getting a camera and connecting through an online interface. Even if this study had not used screen sharing, issues discussed in the first three findings would have still existed. Specifically, communication mediated by technology means it is important for all parties involved to be able to use the shared technologies. The fact that mediated interviews are conducted in people's homes means that privacy issues must be considered. Finally, anytime the interviewer and interviewee are not co-present, details that happen offscreen will be missed.

What is specifically unique in this study is that since we needed screen sharing to collect data around the annotation task, we also collected method data around this practice. This is something missing from existing research handbooks discussing online interviewing and observations. Working through the challenges of screen sharing practice allowed us to reveal more nuanced findings around the technology challenges, as well as elaborate on the differences when conducting think-aloud protocols, and what it means to be on camera, and literally "in someone's face." These findings should provide useful strategies for researchers faced with using these methods, something likely to continue growing well past the COVID-19 pandemic.

5.2.1 Contributions to research methods theory. A common view on how to begin qualitative research is to enter the field as a blank slate, meaning, that the researcher should be open to allowing new ideas to emerge and not be bound to existing literature [27, 31]. This openness to new ideas is key when interpreting qualitative findings, but as other IS scholars have noted, having knowledge of the literature is quite important [31]. This is especially key when trying to allow for new insights to emerge when the cues are reduced in mediated contexts. In our case, balancing this openness with knowledge of past research allowed us to identify the specific differences between online and in person qualitative methods, as well as what makes screen sharing an especially complex task.

Furthermore, this study found that a deep knowledge of literature, technology being used by all parties, and research methods is essential to design an effective mediated research study. Understanding the context (in this case using a computer in a home) of the interview environment is also important. This helps researchers anticipate "things" that might be missed by relying exclusively on what is captured on the video. Actively asking the participants about what is missing from view can help researchers fill some of the missing context. 


\section{Practical Outcomes for IS Community}

In addition to providing a theory- and empiricallyinformed methodological contribution, this paper offers a set of practical outcomes helpful for the IS community. One way to provide broader implications of research is to translate the academic findings into materials helpful for training the next generation of researchers. To accomplish this goal, people reading this paper have access to two online training videos focused on helping researchers use current video tools to continue their research, even when it is not possible to be physically present.

The first video, Meeting Research Participants Online https://youtu.be/g1pW-BubO88, provides an overview of how to contact, schedule, and explain the technical participation requirements to participants who will be part of a mediated research study. The video discusses screen sharing, using multiple researchers, and the importance of conducting multiple interviews with the same participants. Choosing scheduling software is particularly important because the interviews occur in people's homes and often outside typical work hours.

The second video, Getting Meaningful Data from Mediated Qualitative Research, https://youtu.be/nT_D9A6gz4M invites researchers to think outside the box when collecting observation and interview data online. The video discusses building trust, and ways to collect more robust data while experiencing mediated conversations. In addition, we discuss how to design an interview schedule, share informed consent documents, and build rapport in a reduced cues environment. Finally, we discuss the value of having two researchers participate in the online interviews.

\section{Conclusion}

Synchronous, mediated communication has become the "new normal" during the COVID-19 pandemic and will likely continue to be an important feature of work after the pandemic resolves. As such, this is an opportune moment to develop strategies for making the most of mediated interviewing and observing for qualitative research. Our study provides a useful contribution to this effort by highlighting challenges we faced when using a mediated thinkaloud protocol and offering solutions for future researchers.

We have also contributed to theoretical development by discussing how cues-filtered-out and surveillance theories apply in the context of interviewing and observing through synchronous video platforms. Our findings suggest that low cues and a panoptic effect are challenges for this type of research, but that the challenges can be overcome through multiple interviews and strategic rapport building.

\section{Acknowledgements}

This work was supported by a grant from the National Science Foundation [award \# 2029692, 2029698, \& 2029719] RAPID/Collaborative Research: Human-AI Teaming for Big Data Analytics to Enhance Response to the COVID-19 Pandemic. Any opinions, findings, and conclusions or recommendations expressed in this material are those of the author(s) and do not necessarily reflect the views of the National Science Foundation. Additionally, the project was supported by The University of Texas Good Systems Bridging Barriers Project supporting interdisciplinary research.

\section{References}

[1] Stephens, K.K., J.L.S. Jahn, S. Fox, et al., "Collective Sensemaking Around COVID-19: Experiences, Concerns, and Agendas for our Rapidly Changing Organizational Lives", Management Communication Quarterly, 2020, pp. 089331892093489.

[2] Xie, B., D. He, T. Mercer, et al., "Global health crises are also information crises: A call to action", Journal of the Association for Information Science and Technology, 2020, pp. asi.24357.

[3] Gregory, K., "Online Communication Settings and the Qualitative Research Process: Acclimating Students and Novice Researchers", Qualitative Health Research 28(10), 2018, pp. 1610-1620.

[4] Janghorban, R., R.L. Roudsari, and A. Taghipour, "Skype interviewing: The new generation of online synchronous interview in qualitative research", International Journal of Qualitative Studies on Health and Well-being 9(1), 2014.

[5] Salmons, J.E., Doing qualitative research online, Sage, 2015.

[6] Culnan, M.J., and M.L. Markus, "Information technologies", In Handbook of organizational communication: An interdisciplinary perspective. Sage Publications, Inc, Thousand Oaks, CA, US, 1987, 420443.

[7] Walther, J.B., "Interpersonal Effects in ComputerMediated Interaction: A Relational Perspective", Communication Research 19(1), 1992, pp. 52-90.

[8] Walther, J.B., "Computer-Mediated Communication: Impersonal, Interpersonal, and Hyperpersonal Interaction", Communication Research 23(1), 1996, pp. $3-43$.

[9] Fielding, N., R.M. Lee, and G. Blank, eds., The SAGE handbook of online research methods, London: SAGE, Los Angeles, 2017. 
[10] Daft, R.L., and R.H. Lengel, "Organizational information requirements, media richness and structural design", Management science 32(5), 1986, pp. 554-571.

[11] Short, J., E. Williams, and B. Christie, The social psychology of telecommunications, John Wiley \& Sons, 1976.

[12] O'Connor, H., and C. Madge, “Online Interviewing”, In The SAGE Handbook of Online Research Methods. SAGE Publications Ltd, 1 Oliver's Yard, 55 City Road London EC1Y 1SP, 2017, 416-434.

[13] Deakin, H., and K. Wakefield, "Skype interviewing: reflections of two $\mathrm{PhD}$ researchers", Qualitative Research 14(5), 2014, pp. 603-616.

[14] Botan, C., "Communication work and electronic surveillance: A model for predicting panoptic effects", Communication Monographs 63(4), 1996, pp. 293-313.

[15] D'Urso, S.C., "Who's Watching Us at Work? Toward a Structural?Perceptual Model of Electronic Monitoring and Surveillance in Organizations", Communication Theory 16(3), 2006, pp. 281-303.

[16] Bjerke, T.N., "When My Eyes Bring Pain to My Soul, and Vice Versa: Facing Preconceptions in Email and Face-to-Face Interviews", Qualitative Health Research 20(12), 2010, pp. 1717-1724.

[17] Burns, E., "Developing Email Interview Practices in Qualitative Research", Sociological Research Online 15(4), 2010, pp. 24-35.

[18] Ison, N.L., "Having their say: email interviews for research data collection with people who have verbal communication impairment", International Journal of Social Research Methodology 12(2), 2009, pp. 161-172.

[19] James, N., and H. Busher, "Credibility, authenticity and voice: dilemmas in online interviewing", Qualitative Research 6(3), 2006, pp. 403-420.

[20] Jowett, A., E. Peel, and R. Shaw, "Online Interviewing in Psychology: Reflections on the Process", Qualitative Research in Psychology 8(4), 2011, pp. 354-369.

[21] Voida, A., E.D. Mynatt, T. Erickson, and W.A. Kellogg, "Interviewing over instant messaging", Extended abstracts of the 2004 conference on Human factors and computing systems - CHI '04, ACM Press (2004), 1344.

[22] Hanna, P., "Using internet technologies (such as Skype) as a research medium: a research note", Qualitative Research 12(2), 2012, pp. 239-242.

[23] Lo Iacono, V., P. Symonds, and D.H.K. Brown, "Skype as a Tool for Qualitative Research Interviews", Sociological Research Online 21(2), 2016, pp. 103-117.

[24] Sullivan, J.R., "Skype: An Appropriate Method of Data Collection for Qualitative Interviews?", 2012, pp. 8.

[25] Nardi, B.A., My life as a night elf priest: an anthropological account of World of warcraft, University of Michigan Press : University of Michigan Library, Ann Arbor, 2010.

[26] Lewis, C., Using the Thinking-Aloud Method in Cognitive Interface Design, Yorktown Heights, NY, 1982.

[27] Glaser, B., and A. Strauss, The Discovery of Grounded Theory: Strategies for Qualitative Research, Aldine Transaction, Chicago, 1967.

[28] Gibson, J.J., The ecological approach to visual perception: classic edition, Psychology Press, 2014.
[29] Stephens, K.K., B.W. Robertson, and D. Murthy, "Throw me a lifeline: Articulating mobile social network dispersion and the social construction of risk in rescue communication":, Mobile Media \& Communication, 2019.

[30] Evans, S.K., K.E. Pearce, J. Vitak, and J.W. Treem, "Explicating Affordances: a Conceptual Framework for Understanding Affordances in Communication Research", Journal of Computer-Mediated Communication 22(1), 2017, pp. 35-52.

[31] Urquhart, C., and W. Fernández, "Using Grounded Theory Method in Information Systems: The Researcher as Blank Slate and Other Myths":, Journal of Information Technology, 2013. 